\title{
Medición de la calidad por medio de Niveles Sigma para monitorear el mejoramiento de procesos organizacionales controlados por ISO 9001
}

\section{Resumen}

\section{Frank N. Delgado-Moreno ${ }^{1}$}

PASCUAL RuEda Forero ${ }^{1}$

Introducción: Las certificaciones con las normas de la calidad ISO 9000 se han incrementado y expandido en Colombia. En consecuencia, estudios han sido realizados por investigadores en diferentes países con el fin de conocer el impacto de la implementación de las normas ISO 9000 en las empresas. Sin embargo, pocos estudios han sido realizados para medir la calidad después de la implementación de estas normas. Objetivo: esta investigación se enfocó a explorar la medida de la calidad de un caso de estudio en Colombia después de la implementación de las normas ISO 9000. Materiales y Métodos: el caso de estudio está representado por una empresa certificada con la norma ISO 9001, la cual poseía el Premio Colombiano a la Calidad de la Gestión y Premio Iberoamericano de la Calidad. El método se basó en enfoque cuantitativo con alcance de tipo exploratorio y diseño de investigación no experimental-transversal con un tipo de muestra no probabilístico-intencional. Resultados: el caso de estudio presentó calidad de incompetencia mundial. Conclusión: la certificación ISO 9001 no aseguró la calidad de los procesos. Se deben implementar técnicas que mejoren la calidad de los procesos de la organización y fortalezcan la implementación de la norma ISO 9001.

Palabras Clave: ISO 9001, Calidad, Aseguramiento de la calidad, Nivel sigma, Ventaja competitiva, Mejoramiento continuo

1 Universidad Santo Tomas de Bucaramanga, Colombia.

Autor de correspondencia: Delgado-Moreno, F. (Frank): Carrera 18 no 9 - 27 Universidad Santo Tomas, Bucaramanga. Teléfono: 6800801 - 3143670569 . Correo electrónico: Frank.delgado@ustabuca.edu.co
Historia del artículo:

Artículo recibido: 30-VI-2017/ Aprobado: 14-XI-2018

Disponible online: 3 de febrero de 2019

Discusión abierta hasta octubre de 2020 


\title{
Quality Measuring Through Sigma Levels for Monitoring Improvement of Organizational Processes Controlled by ISO 9001
}

\begin{abstract}
Introduction: Colombia has noticed the growth and expansion of certified companies under ISO 9000 standards. Consequently, numerous studies have been conducted to determine the impact of the implementation in companies. However, few studies have been conducted to measure the quality after ISO 9000 deployment. Objective: This research is aimed at exploring the measure of the quality of companies in Colombia after implementation of Quality Management System ISO 9000. Materials and Methods: The case study is represented for a certified company under ISO 9001 standard, which it had won the Colombian Prize for Quality Management and Ibero-American Quality Award. The case study was developed through a quantitative approach, an exploratory scope and non-experimental-transverse research design with a non-probabilistic-intentional sample type. Results: The case study presented quality of global incompetence. Conclusion: ISO 9001 certification did not guarantee the quality of the processes. Techniques that improve the quality of the organization's processes and strengthen the implementation of ISO 9001 standards should be implemented.
\end{abstract}

Keywords: ISO 9001, Quality, Quality assurance, Sigma level, Competitive advantage, Continuos improvement.

\section{A medição da qualidade através de níveis Sigma para monitorar o melhoramento de processos organizacionais controlados por ISO 9001}

\section{Resumo}

Introdução: As certificações com normas ISO 9000 da qualidade têm aumentado e ampliado na Colômbia. Consequentemente, numerosos estudos foram realizados por pesquisadores em diferentes países, a fim de conhecer o impacto da implementação das normas ISO 9000 em empresas. Entretanto, poucos estudos têm sido realizados para medir a qualidade após a aplicação destas normas. Objetivo: Esta pesquisa focada explorar a extensão e garantia de qualidade de um estudo de caso na Colômbia após a implementação da ISO 9000. Materiais e Métodos: Um estudo de caso é representado por uma empresa certificada ISO 9001, que tinha o Prêmio colombiano de Gestão da Qualidade eo Prêmio Ibero-americano da Qualidade. 0 método foi baseada em quantitativa exploratória âmbito abordagem de pesquisa e não reticulado delineamento experimental com um tipo de amostra não probabilística intencional. Resultados: 0 estudo de caso apresentou qualidade de incompetência global. Conclusão: ISO 9001 não garante a qualidade dos processos estudados. Técnicas devem ser implementadas para melhorar a qualidade dos processos organizacionais e fortalecer a implementação da norma ISO 9001.

Palavras-chave: ISO 9001, Qualidade, Asseguramiento de qualidade, Nível sigma, Vantagem competitiva, Aperfeiçoamento contínuo. 


\section{Introducción}

La Organización Internacional de Normalización (International Standard Organization) o ISO por sus siglas en inglés, describe en la Norma ISO 9000:2015 lo siguiente: "Calidad es el grado en que el conjunto de características inherentes de un objeto cumple con los requisitos" (p. 22). Asimismo, la norma describe que el aseguramiento de la calidad significa: "Parte de la norma orientada a proporcionar confianza en que se cumplirán los requisitos de la calidad" (p.19). En consecuencia, muchas empresas en Colombia se han certificado con las normas ISO 9001. La Figura. 1 muestra el crecimiento de las empresas certificadas en Colombia.

Sin embargo, investigadores como Hurtado, Rodríguez, Fuentes y Galleguillos (2009) sostienen:

La certificación en ISO 9000 no garantiza un producto o servicio de calidad, solamente asegura que una empresa llevará a cabo una serie de procedimientos los cuales controlaran dicha calidad, por lo cual tampoco asegura que la empresa trabajando bajo este sistema necesariamente tendrá éxito en el mercado en el cual se desarrolla (p. 24).

Igualmente, otros investigadores que han abarcado el tema concluyeron que las normas ISO 9000 no garantizan un producto de calidad (Hill, 1996; Zhu y Sheuermann, 1999). Rodríguez y González (2003) estudiaron 258 empresas en España, y concluyeron que las normas ISO 9000 no mejoran los costes, la producción ni los beneficios comerciales. Hurtado, Rodríguez, Fuentes y Galleguillos (2009) concluyeron que la implementación de las normas ISO 9000 no representa calidad ni mejoras financieras, solamente, adherirse a un sistema de calidad y certificarse. Anderson et al. (1999) concluyeron que la ventaja competitiva es muy pequeña y que los beneficios en ingresos se disipan rápidamente.

Por otra parte, Joubert (1998) concluyó que el Sistema de Gestión de la Calidad está enfocado a lograr únicamente la certificación, pero no a lograr el mejoramiento continuo, lo cual crea una falsa expectativa en obtener ventaja competitiva con respecto a otras empresas.

Figura 1. Crecimiento del número de empresas certificadas con ISO 9001 en Colombia

Fuente: International Organization for Standardization (s.f.)

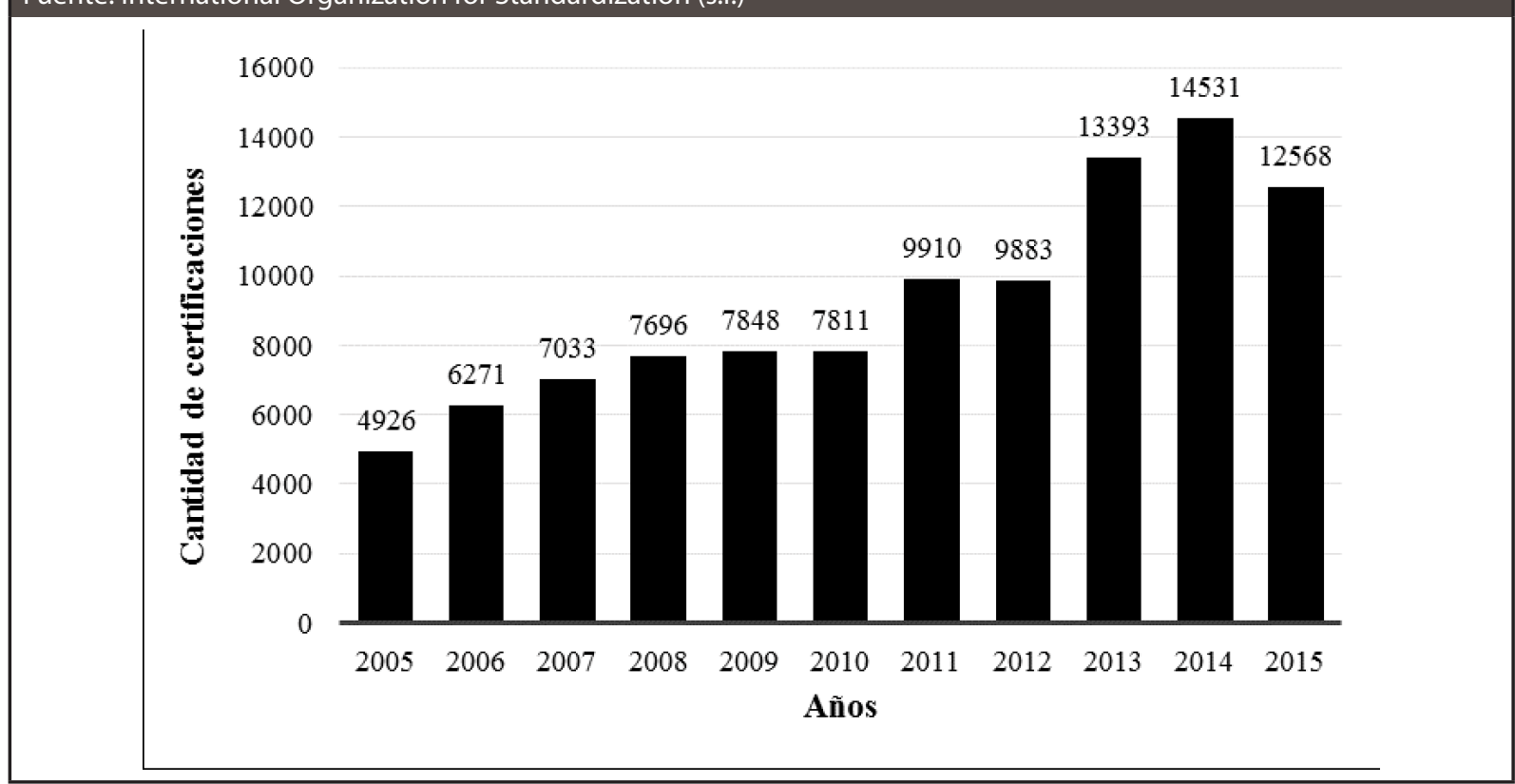


Wayhan et al. (2002) concluyeron que las normas ISO 9000 no otorgan ni calidad ni mejoras financieras. Solamente, adherirse a un sistema de calidad. Morris et al. (2000) concluyeron que la certificación ofrece poca garantía de desempeño efectivo. Gómez, Fontalvo y Vergara (2013) indican que no todas las empresas reciben beneficios de la aplicación de las normas ISO 9000, estos beneficios dependen de la rigurosidad de la aplicación de las mismas. Goedhuys y Sleuwaegen (2013) encontraron que las mejoras productivas después de ISO 9000 se encontraban más predominantes en mercados menos desarrollados. Sumaedi y Yarmen (2015) indican que la efectividad de ISO 9000 es desconocida, sobre todo, en sectores de manufactura de productos alimenticios donde no existe aún un elemento de medición de la efectividad, el cual haya sido ampliamente aceptado. Igualmente, Heras y Borail (2015) determinan que ISO 9000 está enfocada a mantener una imagen corporativa y no a la efectividad interna de los procesos.

Por otra parte, otros investigadores como Terziovski, Samson y Dow (1997) concluyeron que los resultados son positivos con el compromiso de la organización y rendimiento operativo. Bayati y Taghavi (2007) concluyeron que las normas mejoran el rendimiento de las empresas certificadas. Hong y Phitayawejwiwat (2005) concluyeron que la certificación con ISO 9001 presenta beneficios en diferentes factores como "liderazgo", "planeación" y "resultados en calidad". Andrea (2016) sostiene que ISO 9001 mejora la satisfacción del cliente en términos de efectividad y reducción de quejas pero tiene un efecto negativo sobre participación, comunicación y reducción de costos.

En Colombia, según Rodríguez (2012), el Centro de Productividad para el Instituto Colombiano de Normas Técnicas (ICONTEC) realizó un estudio desde el año 2000 al 2007 donde evaluaron 463 empresas de diferentes tipos: pequeñas, medianas y grandes en las ciudades de Bogotá, Cali, Medellín y Bucaramanga. Algunos de los factores evaluados fueron los siguientes: presupuesto, ventas, exportaciones, satisfacción al cliente y atributos de la calidad. El resultado del es- tudio del ICONTEC mostró que 95\% de los encuestados manifestó impacto positivo en las empresas.

Por otra parte, Marín (2014) manifiesta impacto positivo de crecimiento en variables económicas como "productividad", "ventas", "activos", "pasivos" y otros. Fajardo, Velasco y Tobón (2012) sostienen que ISO 9000 no tuvo impacto positivo ni en la estructura organizativa ni en la innovación. Osorio, Cruz y Romero (2015) concluyó que el impacto fue positivo en calidad y competitividad. Bohórquez (2010) concluyó que la certificación en ISO 9000 no es una ventaja competitiva para la organización.

Asimismo, de las Casas y Convers (2015) concluyeron que el impacto fue positivo en factores como "liderazgo", "planeación de la calidad", "auditoria y evaluación de la calidad", "diseño del producto", "gestión de la calidad del proveedor", "control y mejoramiento del proceso" etcétera. Rodríguez y González (2003) analizaron 131 empresas con 15 ventajas (reglas, documentación, proveedores, motivación, comunicación, formación, productividad, costes, auditoria, clientes, cuota de mercado, acceder a otros mercados, diferenciar la empresa y mejorar la imagen), las cuales fueron seleccionadas de la literatura de la certificación en ISO 9000. Los resultados indican que solo las ventajas: reglas, documentación, proveedores, formación y auditoría cumplieron con las necesidades de las empresas. La Tabla 1 resume los objetos de estudio y los resultados de estas investigaciones.

La Tabla 1 muestra que sólo seis estudios fueron enfocados a medir la calidad después de la implementación de ISO 9000. Además, se observa que el 57\% de los estudios presentó impacto (-) sobre resultados de objetos diversos. Adicionalmente, se observa que sólo tres estudios en Colombia tenían como objeto los niveles de calidad. En consecuencia, lo anterior indica que las conclusiones de los estudios continúan dividas respecto al impacto positivo o negativo de la implementación de ISO 9000. Por ejemplo, en Colombia se han desarrollado algunos estudios para determinar el impacto de la implementación de las normas ISO 9000 y certificación ISO 9001 (Tabla 2). 
TABLA 1. ALGUNOS ESTUDIOS REALIZADOS SOBRE EL IMPACTO DE ISO 9000

\begin{tabular}{|c|c|c|c|c|c|c|}
\hline \multirow{2}{*}{ Autores } & \multicolumn{2}{|c|}{ Objetos } & \multicolumn{2}{|c|}{ Impacto } & \multicolumn{2}{|c|}{ País } \\
\hline & Otros & La calidad & $(+)$ & $(-)$ & Col. & Otros \\
\hline Rodríguez-Escobar, J.A.; González-Benito, J. (2003). & 11 & & & $(-)$ & & $\mathrm{x}$ \\
\hline Hill, S. (1996). & & $\mathrm{x}$ & & $(-)$ & & $\mathrm{x}$ \\
\hline Zhu, Z.; Sheuermann, L. (1999). & & $\mathrm{x}$ & & $(-)$ & & $\mathrm{x}$ \\
\hline Hurtado, R.; Rodríguez, R.; Fuentes, H.; Galleguillos, C. (2009). & & & & $(-)$ & & $\mathrm{x}$ \\
\hline Anderson, S.; Daly, D.; Johnson, M. (1999). & 1 & & & $(-)$ & & $\mathrm{x}$ \\
\hline B. Joubert, 1998. & 1,3 & & & $(-)$ & & $\mathrm{x}$ \\
\hline Wayhan, V.; Kirche, E.; Khumawhala, B. (2002). & & $x$ & & $(-)$ & & $\mathrm{x}$ \\
\hline Morris, A.; Crawford, J.; Carter, D.; Mazotta, F. (2000) & 4 & & & $(-)$ & & $\mathrm{x}$ \\
\hline Terziovski, M.; Samson, D.; Dow, D. (1997). & 5,6 & & $(+)$ & & & $\mathrm{x}$ \\
\hline Bayati, A.; Taghavi, A. (2007). & 7 & & $(+)$ & & & $\mathrm{x}$ \\
\hline Hong, J.; Phitayawejwiwat, S. (2005). & 8 & & $(+)$ & & & $\mathrm{x}$ \\
\hline Rodríguez-Fonseca, F. (2012). & 9 & & $(+)$ & & $\mathrm{x}$ & $\mathrm{x}$ \\
\hline Casadesús, G.; Marti, G.; Heras, I. (2001). & * & & $(+)$ & & & $\mathrm{x}$ \\
\hline Bondarenko-Pisemskaya, N. (2007). & * & & & & & $\mathrm{x}$ \\
\hline Morelos-Gómez, j.; Fontalvo, T.; Vergara, T. (2013). & 2 & & $(+)$ & $(-)$ & $\mathrm{x}$ & \\
\hline Goedhuys, M; Sleuwaegen, L. (2013). & 11 & & $(+)$ & $(-)$ & & $\mathrm{x}$ \\
\hline Sumaedi S.; Yarmen, M. (2015). & 13 & & & $(-)$ & & $\mathrm{x}$ \\
\hline Heras-Saizarbitoria, I.; Borail, O. (2015). & 13 & & & $(-)$ & & $\mathrm{x}$ \\
\hline Storz, C. (2007). & * & & $(+)$ & $(-)$ & & $\mathrm{x}$ \\
\hline Ming-Ong, O.; Kathawala, Y.; Sawalha, N. (2015). & * & & $(+)$ & & & $\mathrm{x}$ \\
\hline Andrea, C. (2016). & $*, 4,9$ & & $(+)$ & $(-)$ & & $\mathrm{x}$ \\
\hline Robles-Marín, V. (2014). & $*, 1$ & & $(+)$ & & $\mathrm{x}$ & \\
\hline Fajardo-Ortiz, M.; Cano-Velasco, L.; Tobón-Gordillo, M. (2012). & * & & & $(-)$ & $\mathrm{x}$ & \\
\hline Osorio-Gómez, J.C.; Cruz-Giraldo, E.V.; Romero-Vega, M.C. (2015). & 14 & $\mathrm{x}$ & $(+)$ & & $\mathrm{x}$ & \\
\hline Bohórquez, L.H. (2010). & * & & & $(-)$ & $x$ & \\
\hline Benzaquen-de las Casas, J.; Convers-Sorza, J.D. (2015). & * & $\mathrm{x}$ & $(+)$ & & $\mathrm{x}$ & \\
\hline $\begin{array}{l}\quad \text { Nota: } 1 \text { = Ventaja competitiva, } 2=\text { Beneficios de ingresos, } 3= \\
=\text { Rendimiento operativo, } 6=\text { Compromisos organizacionales, } 7= \\
\text { satisfacción del cliente y exportaciones; } 10=\text { Reglas, documentacic } \\
\text { oroductivas, } 12 \text { = Costes, producción y beneficios comerciales; } 13 \\
\text { Col. = Colombia. Fuente: Autor. }\end{array}$ & $\begin{array}{l}\text { ejorami } \\
\text { dimien }\end{array}$ & $\begin{array}{l}\text { nto continu } \\
8=\text { Lidera }\end{array}$ & $4=$ & d, * & $\begin{array}{l}\text { peño } \\
\text { ción, } \\
\text { os; } 11 \\
=\text { Otrc }\end{array}$ & $\begin{array}{l}\text { fectivo, } \\
=\text { Ventas } \\
\text { Mejora } \\
\text { objetos }\end{array}$ \\
\hline
\end{tabular}

La Tabla 2 muestra los estudios representativos en Colombia realizados para determinar el impacto de las normas ISO 9000. Se observa que en algunos casos los resultados fueron positivos y otros negativos. Igualmente, se observa que el método comúnmente utilizado fue "la encuesta", la cual no fue la misma para los diferentes estudios. Por otra parte, se observa que el estudio realizado por Bohórquez (2010) indica que fueron medidas todas las empresas y el resultado fue negativo. De igual forma, se observa que sólo tres de estos estudios se enfocaron a evaluar la calidad (Centro 
Nacional de Productividad, s.f.; Osorio, Cruz y Romero, 2015; Benzaquen y Convers, 2015), los cuales presentaron impacto positivo. Por otra parte, estos tres estudios utilizaron encuestas como métodos de medición. Las encuestas no representan exactitud sobre los resultados de la implementación debido a que dependen de la opinión de los encuestados que en muchos casos son los gerentes y personal involucrado en el proceso. Heras, Aranaa y Boiral (2015, p. 139) sostienen: "Los resultados de las encuestas tienden a ser influenciados por la libre presentación de informes y el sesgo de deseabilidad social relacionada con la posición de los encuestados".

TABLA 2. PRINCIPALES ESTUDIOS EN COLOMBIA REALIZADOS ENTRE 2006 Y 2015 SOBRE EL IMPACTO DE ISO 9000

\begin{tabular}{|c|c|c|c|c|c|}
\hline Autor & Sector & Método & \# & Criterios medidos & I \\
\hline $\begin{array}{l}\text { Centro Nacional de } \\
\text { Productividad (s.f.). }\end{array}$ & Diferentes & Encuestas & 563 & $\begin{array}{l}\text { Mejoramiento de la calidad, incremento de la } \\
\text { productividad, el desarrollo de su capacidad } \\
\text { competitiva, control de la calidad, capacitación } \\
\text { de personal, comportamiento de las ventas, } \\
\text { exportaciones, satisfacción del cliente, impacto } \\
\text { con relación a los ocho principios de la calidad } \\
\text { y atributos de la calidad. }\end{array}$ & $(+)$ \\
\hline $\begin{array}{l}\text { Rodríguez-Fonseca, } \\
\text { F. (2012). }\end{array}$ & Diferentes & $\begin{array}{l}\text { Revisión de } \\
\text { literatura }\end{array}$ & 1 & $\begin{array}{l}\text { Marketing, finanzas, competitividad, } \\
\text { eficiencia productiva y de satisfacción de los } \\
\text { clientes. }\end{array}$ & $(+)$ \\
\hline $\begin{array}{l}\text { Morelos-Gómez, } \\
\text { j.; Fontalvo, T.; } \\
\text { Vergara, T. (2013). }\end{array}$ & Diferentes & $\begin{array}{l}\text { Reporte } \\
\text { de estados } \\
\text { financieros }\end{array}$ & 25 & $\begin{array}{l}\text { Margen bruto, margen operacional, margen } \\
\text { neto. Razón de utilidad bruta/valor agregado. } \\
\text { Productividad de capital. Razón utilidad } \\
\text { operativa/capital de trabajo. Razón utilidad } \\
\text { neta/capital de trabajo. }\end{array}$ & $(+)$ \\
\hline $\begin{array}{l}\text { Robles-Marín, V. } \\
\text { (2014). }\end{array}$ & Diferentes & Encuestas & 400 & Activos, pasivos, ventas, etc. & $(+)$ \\
\hline $\begin{array}{l}\text { Fajardo-Ortiz, M.; } \\
\text { Cano-Velasco, L.; } \\
\text { Tobón-Gordillo, M. } \\
\text { (2012). }\end{array}$ & Agroindustrial & Encuestas & 3 & Estructura organizativa e innovación. & $(-)$ \\
\hline $\begin{array}{l}\text { Osorio-Gómez, J.C.; } \\
\text { Cruz-Giraldo, E.V.; } \\
\text { Romero-Vega, M.C. } \\
\text { (2015). }\end{array}$ & Salud & Encuestas & 37 & Calidad y productividad. & $(+)$ \\
\hline $\begin{array}{l}\text { Bohórquez, L.H. } \\
\text { (2010). }\end{array}$ & Diferentes & $\begin{array}{l}\text { Empresas } \\
\text { certificadas/Pib }\end{array}$ & Todas & $\begin{array}{l}\text { Desarrollo económico, eficiencia } \\
\text { gubernamental, eficiencia en los negocios por } \\
\text { empresas del país e infraestructura. }\end{array}$ & $(-)$ \\
\hline $\begin{array}{l}\text { Benzaquen-de las } \\
\text { Casas, J.; Convers- } \\
\text { Sorza, J.D. (2015). } \\
\text { las Casas y J. } \\
\text { Convers-Sorza, } \\
2015\end{array}$ & Diferentes & Encuestas & 6000 & $\begin{array}{l}\text { Liderazgo, planeación de la calidad, auditoría y } \\
\text { evaluación de la calidad, diseño del producto, } \\
\text { gestión de la calidad del proveedor, control } \\
\text { y mejoramiento del proceso, educación } \\
\text { y entrenamiento, círculos de la calidad y } \\
\text { enfoque hacia la satisfacción del cliente. }\end{array}$ & $(+)$ \\
\hline
\end{tabular}


Por las anteriores razones, se observa que tanto a nivel internacional como en Colombia, no está resuelto si la implementación de las normas ISO 9000 y la respectiva certificación ISO 9001, aseguran la calidad de las empresas. Por otra parte, son pocos los estudios enfocados a medir la calidad después de la implementación de las normas ISO 9000 (Tabla 1 y 2). Los pocos estudios que existen en Colombia han sido realizados por medio de encuestas que tienden a ser influenciadas y sesgadas (Heras, Aranaa y Boiral, 2015). Por esta razón, también se desconoce la calidad resultado después de la implementación de las normas ISO 9000 y certificación ISO 9001.

El propósito de este artículo es explorar la calidad de los procesos relacionada a los defectos, los cuales causan altos costos debido a las fallas internas de las empresas (Gracia, López, 2007). Estos defectos no son evidentes - como se observa en los resultados de las (Tabla 1 y 2 ) - debido a la errónea interpretación de las empresas que cuando alcanzan la certificación ISO 9001 se basan en la falsa creencia de haber alcanzado niveles de seguridad que les otorga ventaja competitiva respecto a otras empresas (Hill, 1996).

El método se basó en enfoque cuantitativo con alcance de tipo exploratorio y diseño de investigación no experimental-transversal con un tipo de muestra no probabilístico-intencional. La medición se realizó durante seis meses. La muestra fue constituida por cuatro procedimientos y un proceso total pertenecientes a un caso de estudio de una empresa certificada con la norma ISO 9001. Además, el caso de estudio poseía el Premio Nacional de la Calidad y Premio Iberoamericano de la Calidad. Los resultados del caso de estudio presentaron calidad de incompetencia mundial para los procedimientos y proceso estudiados.

\section{Método}

El enfoque de la investigación fue cuantitativo con alcance de tipo exploratorio. No se definieron hipótesis para este tipo de alcance (Hernández,
Fernández, Baptista, 1996). El diseño de la investigación fue no experimental - no se crearon condiciones de manipulación. Se les observó de manera naturalista- de tipo transversal exploratoria.

La unidad de análisis fue constituida por procesos controlados de empresas certificadas con ISO 9001. La población fue delimitada a los procesos de empresas en Santander (Colombia), las cuales pertenecían a cualquier sector industrial con cantidad de empleados superior a 200. Las empresas contaban con más de 10 años desde la fundación, y presentaban certificación ISO 9001 entre junio de 2015 y julio de 2016. Hernández, Fernández, Baptista (1996, p. 174) sostienen: “Una población es el conjunto de todos los casos que concuerdan con una serie de especificaciones".

La muestra - seleccionada desde la población- fue no probabilística o dirigida (Hernández, Fernández y Baptista, 1996) de tipo intencional (Padua, 1979, citado en Kazes, 2009) y por conveniencia. "Este tipo de muestra representa los casos seleccionados a criterio del experto" (Sampieri, 2010, p. 401). El tamaño de la muestra correspondió a un único caso que corresponde a un tamaño de muestra explicativa - discutidora de teoría- tipo 5 (O'Rourke, 2005). Este tamaño de muestra representa: "el análisis de caso como puesta a prueba de una determinada proposición" (Lipjphart citado en Kazes, 2009, p. 77).

El caso de estudio corresponde a un proceso de apoyo controlado por la norma ISO 9001 de una empresa certificada con la misma norma. La selección de la empresa se realizó de acuerdo a los criterios de selección de muestra explicados en esta sección - muestra no probabilística o dirigida de tipo intencional (Padua, 1979, citado en Kazes, 2009) y por conveniencia (Hernández, Fernández, Baptista, 2010, p. 401)-, el proceso fue seleccionado teniendo en cuenta el principio de la "Gestión por Procesos" recogido en la Gestión de Calidad Total, en las normas ISO 9000 y el Modelo de la Fundación Europea para la Gestión de la Calidad (European Foundation for Quality Management) o EFQM por sus siglas 
en inglés (Camisón, Cruz y González, 2006). Este principio expresa lo siguiente:

La gestión por procesos es una práctica que consiste en gestionar integralmente cada uno de los procesos que tienen lugar en la empresa, y no únicamente los procesos productivos o relativos al área de ventas, como tradicionalmente se ha venido haciendo (p. 863).

Para la selección del proceso también se basó en los conceptos de muestra de tipo intencional (Kazez, 2009) y por conveniencia (Hernández, Fer- nández, Baptista, 2010). El proceso seleccionado del caso de estudio fue identificado como el proceso de "facturación de los servicios médicos prestados a los pacientes". Las facturas eran la salida de este proceso, la cual era recibida por el proceso de "Radicación de la factura" (Figura 2)

Las variables que se midieron fueron datos de tipo categórico. Estas variables fueron las "Facturas defectuosas", lo cual significó que si la factura tenía algún defecto en el formato o en los anexos entonces era rechazada. La selección de esta variable se basó en el criterio de "costos por fallas internas" (Gracia, López, 2007) en el proceso seleccionado.

\section{Figura 2. Proceso seleccionado "Facturación de servicios médicos prestados a pacientes"}

\section{Fuente: Autor}

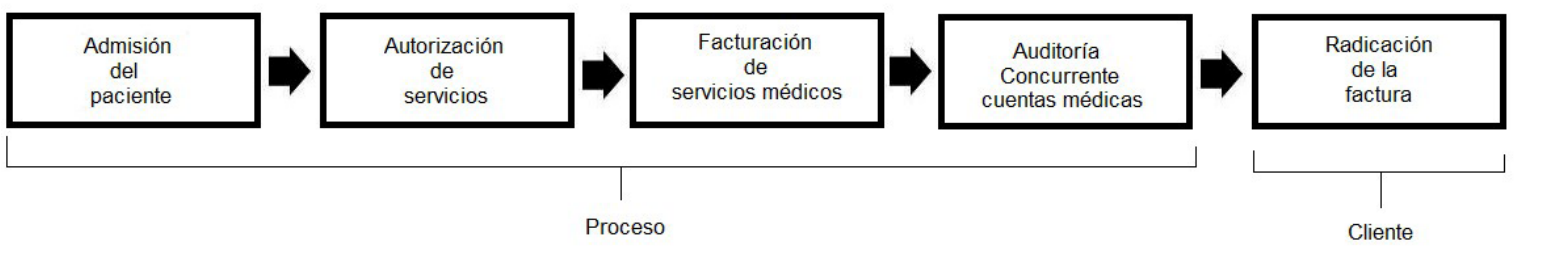

\section{Figura 3. Valores monetarios no recibidos por facturas defectuosas}

Fuente: Autor

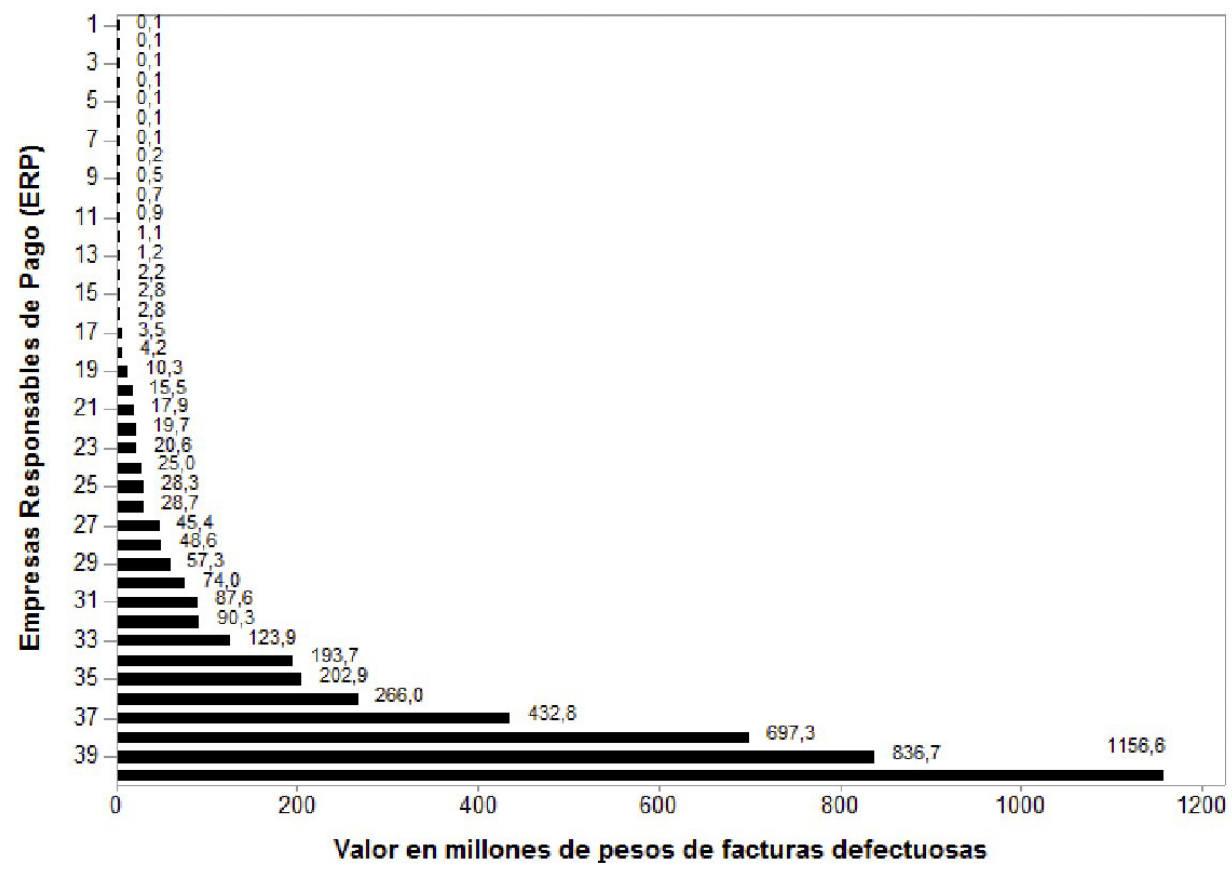


La Figura 3 muestra que el valor monetario no recibido por "Facturas defectuosas" correspondió a 4.526 millones de pesos, según las facturas devueltas por las Empresas Responsables de Pago (ERP) durante seis meses. A la izquierda se observa un número asignado a cada ERP. Éstos están ordenados de menor a mayor valor con respecto a la "Factura defectuosa".

La recolección de los datos de las variables se realizó a través de bases históricas correspondientes a seis meses. El procedimiento de recolección fue consecutivo de acuerdo a las fechas de expedición de las facturas. El tamaño de los datos fue de
17.128 facturas revisadas y se contabilizaron 1.956 facturas devueltas por defectuosas. Las oportunidades para la realización de un defecto en cada factura se muestran en la Tabla 3.

En la Tabla 3 se muestra diferentes tipos de defecto desde A hasta G, y en la sección derecha se contabilizó la cantidad de oportunidades de cometer un defecto. La medición de los datos se realizó por medio de las "puntuaciones Z" (Hernández, Fernández, Baptista, 2010), las cuales se compararon con una tabla estandarizada de "niveles sigma" (Tabla 4) para conocer el significado del resultado de la medida calculada con respecto a la calidad.

\section{TABLA 3. IDENTIFICACIÓN DE LOS DEFECTOS EN LAS FACTURAS}

\begin{tabular}{|c|c|c|}
\hline Defectos tipo & Descripción del defecto & Oportunidad \\
\hline \multirow{3}{*}{ A } & Factura con responsable de pago & 1 \\
\hline & Factura con valor a pagar & 2 \\
\hline & Factura con conceptos de servicio & 2 \\
\hline \multirow{2}{*}{ B } & Factura con sello & 1 \\
\hline & Factura con firma de la persona que elabora la factura & 1 \\
\hline \multirow{2}{*}{$\mathbf{C}$} & Factura con firma del usuario & 1 \\
\hline & Factura con detalle de cargos & 2 \\
\hline \multirow{2}{*}{$\mathbf{D}$} & Factura con orden médica original & 1 \\
\hline & Factura con orden médica fotocopiada & 1 \\
\hline \multirow{2}{*}{$\mathbf{E}$} & Factura con autorización original & 1 \\
\hline & Factura con evolución médica & 1 \\
\hline \multirow{2}{*}{$\mathbf{F}$} & Factura con recibo de pago de los copagos & 2 \\
\hline & Factura con original de bonos según entidad & 1 \\
\hline G & Factura con resultados de exámenes gestionados en consulta externa & 2 \\
\hline Total & & 19 \\
\hline
\end{tabular}

\section{TABLA 4. RELACIÓN DE NIVELES SIGMA Y LA CALIDAD}

\begin{tabular}{c|c|c} 
Nivel Sigma & DPMO & Costo de Calidad \\
\hline 2 & 308.357 - Compañías no competitivas & No aplicable \\
\hline 3 & 66.807 & $25-40^{*}$ \\
\hline 4 & 6210 - Promedio de la Industria & $15-25^{*}$ \\
\hline 5 & 233 & $5-15^{*}$ \\
\hline 6 & 3,4 Clase mundial & $<1^{*}$ \\
\hline
\end{tabular}

Nota: ${ }^{*}=\%$ de las ventas. Fuente: O'Rourke, 2005. 
La Tabla 4 muestra que el nivel sigma más deficiente es el nivel 2 (Compañías no competitivas), y el nivel óptimo es el nivel 6 (Clase mundial). El medio de recolección de los datos se realizó con base en un formato de recolección de datos (Tabla 5).

La Tabla 5 muestra un ejemplo de formato para la recolección de las "Facturas defectuosas" encontrados en el proceso. Por otra parte, la preparación de los datos se realizó de la siguiente forma: a) revisión de los datos, b) ajuste de los datos (en caso de que el total - de la Tabla 5- no coincida con la frecuencia de defectos), c) digitación de los datos en Excel, d) Ingreso de los datos en software estadístico (El software estadístico utilizado fue Minitab versión 17).

\section{Procedimiento}

El procedimiento para la medición de la calidad del caso de estudio se realizó de la siguiente for- ma: el equipo de personas asistió aleatoriamente a la empresa durante 6 meses. Cada día revisaban un número consecutivo de facturas - por fecha-, se observaba el defecto por el cual había sido rechazada la factura, y se procedía a completar el formato de la Tabla 5, el cual estaba previamente diseñado en el software Excell. Una vez revisada la cantidad de facturas correspondientes a seis meses, se procedió a obtener las cantidades resultados de facturas defectuosas y a exportar el archivo al software Minitab versión 17. Posteriormente, se determinó el tipo de distribución de los datos, se calculó la puntuación Z -la determinación del tipo de distribución de datos y el cálculo de puntuaciones $\mathrm{Z}$ es ampliamente conocido. Por esta razón, no se describen (Mari, 2013) - de los datos y se comparó los resultados de la puntuación Z con la Tabla de niveles sigma (Tabla 4). "1.5 es el factor de corrección para tener el equivalente de un estudio de largo plazo a corto plazo y viceversa" (Escalante, 2008, p. 244).

\begin{tabular}{|c|c|c|c|}
\hline Defectos tipo & Descripción del defecto & $\begin{array}{c}\text { Facturas } \\
\text { devueltas }\end{array}$ & $\begin{array}{c}\text { Total facturas } \\
\text { devueltas }\end{array}$ \\
\hline \multirow{3}{*}{ A } & Factura con responsable de pago & \# & \multirow{3}{*}{ \# } \\
\hline & Factura con valor a pagar & \# & \\
\hline & Factura con conceptos de servicio & \# & \\
\hline \multirow{2}{*}{ B } & Factura con sello & \# & \multirow{2}{*}{ \# } \\
\hline & Factura con firma de la persona que elabora la factura & \# & \\
\hline \multirow{2}{*}{$\mathbf{C}$} & Factura con firma del usuario & \# & \multirow{2}{*}{$\#$} \\
\hline & Factura con detalle de cargos & \# & \\
\hline \multirow{2}{*}{ D } & Factura con orden médica original & \# & \multirow{2}{*}{$\#$} \\
\hline & Factura con orden médica fotocopiada & \# & \\
\hline \multirow{2}{*}{$\mathbf{E}$} & Factura con autorización original & \# & \multirow{2}{*}{ \# } \\
\hline & Factura con evolución médica & \# & \\
\hline \multirow{2}{*}{$\mathbf{F}$} & Factura con recibo de pago de los copagos & $\#$ & \multirow{2}{*}{ \# } \\
\hline & Factura con original de bonos según entidad & $\#$ & \\
\hline G & $\begin{array}{l}\text { Factura con resultados de exámenes gestionados en consulta } \\
\text { externa }\end{array}$ & $\#$ & $\#$ \\
\hline Total & & 19 & 1956 \\
\hline
\end{tabular}

Nota: cada defecto es igual a una factura defectuosa. Al primer defecto encontrado, la factura era desechada por la ERP. \# = cantidad. Fuente: Autor 


\section{Resultados}

La cantidad de "Facturas defectuosas" (variable categórica) relacionada con los tipos de defectos se muestra en la Tabla 6.

La Tabla 6 muestra que los tipos de defectos A, B y C presentaron la mayor frecuencia. Esta frecuencia estaba relacionada a los procedimientos como se observa en la Figura 3.
La Figura 3 muestra que la cantidad de defectos relacionada a los tipos A, B y C era producida en el procedimiento de "Admisión de paciente" con 1.251 "Facturas defectuosas". Igualmente, muestra la cantidad de "Facturas defectuosas" de acuerdo a cada procedimiento. Conocidos la cantidad de "Facturas defectuosas" producida por cada procedimiento. Se verificó la distribución de cada procedimiento y del proceso total (Tabla 7).

\section{TABLA 6. TOTAL DE "FACTURAS DEFECTUOSAS" RELACIONADAS CON LOS DEFECTOS \\ FUENTE: AUTOR}

\begin{tabular}{|c|c|c|c|}
\hline Defectos tipo & Descripción del defecto & $\begin{array}{l}\text { Facturas } \\
\text { devueltas }\end{array}$ & $\begin{array}{l}\text { Total facturas } \\
\text { devueltas }\end{array}$ \\
\hline \multirow{3}{*}{ A } & Factura con responsable de pago & 95 & \multirow{3}{*}{445} \\
\hline & Factura con valor a pagar & 225 & \\
\hline & Factura con conceptos de servicio & 125 & \\
\hline \multirow{2}{*}{ B } & Factura con sello & 253 & \multirow{2}{*}{443} \\
\hline & Factura con firma de la persona que elabora la factura & 190 & \\
\hline \multirow{2}{*}{$\mathbf{C}$} & Factura con firma del usuario & 88 & \multirow{2}{*}{363} \\
\hline & Factura con detalle de cargos & 275 & \\
\hline \multirow{2}{*}{$\mathbf{D}$} & Factura con orden médica original & 62 & \multirow{2}{*}{327} \\
\hline & Factura con orden médica fotocopiada & 265 & \\
\hline \multirow{2}{*}{$\mathbf{E}$} & Factura con autorización original & 63 & \multirow{2}{*}{188} \\
\hline & Factura con evolución médica & 125 & \\
\hline \multirow{2}{*}{$\mathbf{F}$} & Factura con recibo de pago de los copagos & 67 & \multirow{2}{*}{159} \\
\hline & Factura con original de bonos según entidad & 92 & \\
\hline G & $\begin{array}{l}\text { Factura con resultados de exámenes gestionados en consulta } \\
\text { externa }\end{array}$ & 31 & 31 \\
\hline Total & & 1.956 & 1.956 \\
\hline
\end{tabular}

Figura 3. Macro-proceso de relación de procedimientos con facturas defectuosas

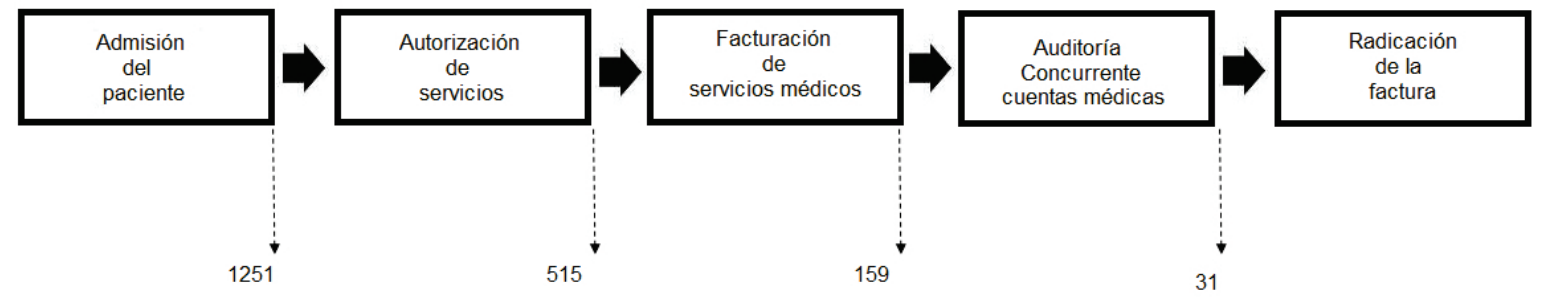


TABLA 7. IDENTIFICACIÓN DEL TIPO DE DISTRIBUCIÓN DE CADA PROCEDIMIENTO Y EL PROCESO TOTAL

\begin{tabular}{c|c|c|c|c|c|c|c|c|c} 
Procedimiento & $\mathbf{p}$ & $\mathbf{q}$ & $\mathbf{n}$ & $\mathbf{y}$ & $\mathbf{m}$ & $\mathbf{s}$ & Condición 1 & Condición 2 & Distribución \\
\hline Procedimiento 1 & 0,073 & 0,93 & 17.128 & 1.251 & $1.250,34$ & 34,05 & $1.250,34$ & $15.877,66$ & Normal \\
\hline Procedimiento 2 & 0,032 & 0,97 & 15.877 & 515 & 508,06 & 22,18 & 508,06 & $15.368,94$ & Normal \\
\hline Procedimiento 3 & 0,010 & 0,99 & 15.362 & 159 & 159,00 & 12,54 & 159,00 & $15.203,00$ & Normal \\
\hline Procedimiento 4 & 0,002 & 1,00 & 15.203 & 31 & 31,00 & 5,56 & 31,00 & $15.172,00$ & Normal \\
\hline Proceso & 0,114 & 0,89 & 17.128 & 1.956 & $1.956,00$ & 41,62 & $1.956,00$ & $15.172,00$ & Normal \\
\hline
\end{tabular}

Nota: Las "Facturas defectuosas" —en su origen — corresponden al tipo de distribución Binomial.

Fuente: Autor

TABLA 8. CÁLCULO DE LAS PUNTUACIONES Z DE LOS PROCEDIMIENTOS Y EL PROCESO TOTAL

\begin{tabular}{c|c|c|c|c|c|c|c|c|c|c}
\multirow{2}{*}{$\begin{array}{c}\text { Procedimiento/ } \\
\text { Proceso }\end{array}$} & Defectuosas & Entradas & Op. & Total Op. & \multicolumn{3}{|c|}{ Largo Tiempo (LT) } & \multicolumn{2}{c}{ Corto Tiempo (CT) } \\
\cline { 6 - 11 } & & & & & Dpo & Dpmo & Z & Dpo & Dpmo & Z CT \\
\hline Procedimiento 1 & 1.251 & 17.128 & 10 & 170.128 & 0,007 & $7.353,28$ & 2,45 & 0,0071 & 7.100 & 3,95 \\
\hline Procedimiento 2 & 515 & 15.877 & 4 & 63.508 & 0,008 & $8.109,21$ & 2,4 & 0,0082 & 8.200 & 3,9 \\
\hline Procedimiento 3 & 159 & 15.362 & 3 & 46.086 & 0,003 & $3.450,07$ & 2,74 & 0,003 & 3.000 & 4,24 \\
\hline Procedimiento 4 & 31 & 15.203 & 1 & 15.203 & 0,002 & $2.039,07$ & 2,87 & 0,0025 & 2.500 & 4,37 \\
\hline Proceso & 1.956 & 17.128 & 19 & 349.299 & 0,006 & $5.599,78$ & 2,51 & 0,006 & 6.000 & 4,01 \\
\hline
\end{tabular}

Nota: Dpo $=$ Defectos por oportunidad, Dpmo = Defectos por millón de oportunidades, Op. $=$ oportunidades

Fuente: Autor

TABLA 9. INTERPRETACIÓN DEL RESULTADO DE LA CALIDAD DEL CASO ESTUDIADO

\begin{tabular}{|c|c|c|c|c|}
\hline Proceso/procedimiento & Z LT & $\mathrm{Z} \mathrm{CT}$ & Calidad a CT & Calidad a LT \\
\hline Procedimiento 1 & 2,45 & 3,95 & ( - ) del promedio & no competitiva \\
\hline Procedimiento 2 & 2,4 & 3,9 & ( - ) del promedio & no competitiva \\
\hline Procedimiento 3 & 2,74 & 4,24 & ( - ) del promedio & no competitiva \\
\hline Procedimiento 4 & 2,87 & 4,37 & ( - ) del promedio & no competitiva \\
\hline Proceso Total & 2,51 & 4,01 & (-) del promedio & no competitiva \\
\hline
\end{tabular}

La Tabla 7 muestra que los procedimientos y el proceso total presentaron una distribución normal debido a que cumplieron con las condiciones 1 y 2 para la aproximación a la Normal de la distribución Binomial (Ecuaciones 1 y 2).

$$
\begin{gathered}
\text { Condición } 1: n^{*} p \geq 5 \\
\text { Condición } 2: n^{*}(1-p) \geq 5
\end{gathered}
$$

Donde $p=y / n$, donde $n$ es facturas revisadas, $y$ es facturas no aprobadas y $p$ es la probabilidad de ocurrencia. Después de verificadas las distribuciones se calculó las puntuaciones Z correspondientes a cada procedimiento y el proceso (Tabla 8)

La Tabla 8 muestra que el nivel sigma más bajo correspondió al procedimiento 2 en corto tiempo (CT) y largo tiempo (LT). Por otra parte, el mayor nivel sigma encontrado correspondió al procedimiento 4. Igualmente, la Tabla muestra las cantidades de Dpo y Dpmo de los respectivos procedimientos y proceso total, los cuales fueron calculados con las Ecuaciones 3 y 4. 


$$
\begin{gathered}
\text { DPO }=\left[\frac{\text { Número de defectos }}{\text { Número de Oportunidades * producción total }}\right. \\
\text { DPMO }=[\mathrm{DPO}]^{*} 10^{6}
\end{gathered}
$$

La Tabla 9 muestra la interpretación de los resultados de la calidad de los procedimientos y del proceso total relacionada a los niveles sigmas clasificados en la Tabla 4.

La Tabla 9 muestra que todos los procedimientos y el proceso total presentaron calidad no competitiva en tiempo largo. El procedimiento 2 mostró el nivel más bajo. Por otra parte, en tiempo corto, Los procedimientos 3, 4 y el proceso total presentaron calidad del promedio mundial. Los procedimientos 1 y 2 presentaron calidad por debajo del promedio mundial.

\section{Discusión}

Son muchos los estudios enfocados a medir diferentes aspectos después de la implementación de las normas ISO 9000. Sin embargo, éstos presentan división en los resultados entre impacto positivo y negativo (Tabla 1). Además, son pocos los estudios enfocados a medir la calidad después de la implementación (Tabla 2). Por otra parte, los pocos estudios que existen en Colombia han sido realizados con encuestas que tienden a ser sesgadas (Heras, Aranaa y Boiral, 2015). Además, los resultados de la implementación varían de contexto a contexto de acuerdo a la rigurosidad y desarrollo del mercado (Osorio, Cruz y Romero, 2015; Fajardo, Cano y Tobón, 2012). Asimismo, la efectividad de ISO 9000 aún es desconocida (Bohórquez, 2010). Por estas razones, Identificar la calidad de los procesos controlados con la certificación ISO 9001 es importante porque permite evidenciar los defectos — que están relacionados con la calidad- en los procesos, los cuales causan altos costos internos y externos a empresa (Gracia, López, 2007). Estos defectos no son evidenciables debido a la errónea interpretación de las empresas cuando alcanzan la certificación ISO 9001. Esta errónea interpretación se basa en la falsa creencia de haber alcanzado niveles de seguridad que les otorga ventaja competitiva con respecto a otras empresas (Hill, 1996).

En este estudio, nosotros exploramos la medida de la calidad de procedimientos y del proceso correspondiente. Los procedimientos y el proceso estaban controlados por la normas ISO 9000 y la empresa estaba certificada con la norma ISO 9001. A través de la medición realizada con DPO y niveles sigma, se identificó que el caso de estudio - en tiempo largo- presentó calidad de incompetencia mundial para todos los procedimientos y el proceso total. Por otra parte - en tiempo corto-, los procedimientos y el proceso total presentaron calidad de promedio mundial.

Los resultados significan que en un millón de oportunidades se producirán 5.599,787 defectos en todo el proceso (Tabla 8). Igualmente, los resultados del mejor procedimiento - "Auditoria concurrente y cuentas médicas"-, significan que se presentarán 2.039,071 defectos en un millón de oportunidades. Lo anterior indica que en ningún procedimiento o proceso hubo aseguramiento de la calidad de acuerdo al concepto de la norma ISO 9000:2015.

Los resultados de este estudio son consistentes con los resultados de Hurtado, Rodríguez, Fuentes y Galleguillos (2009); Anderson, Daly y Johnson (1999) con respecto al impacto negativo de la certificación ISO 9001 del objeto de análisis, la calidad. Igualmente, es consistente - en general - con los resultados negativos de Hill (1996); Zhu y Sheuermann (1999); Rodríguez y González (2003); Joubert (1998). Sin embargo, las diferencias de estos estudios con respecto a la presente investigación son el tamaño de muestra y el método. El tamaño de muestra para los estudios citados permitía resultados concluyentes, pero el método estuvo basado en encuestas de percepción, las cuales son no confiables por el sesgo de deseabilidad social relacionada con los encuestados (Osorio, Cruz y Romero, 2015). Por otra parte, esta investigación se basó en un tamaño de muestra exploratoria de un caso de estudio que 
no permite un resultado concluyente, pero se utilizó el método estadístico de puntuaciones Z (Hernández, Fernández, Baptista, 1996) —ampliamente conocido y aceptado-. A diferencia de los otros métodos -encuestas sobre la percepción de personas-, este método estadístico fue aplicado sobre procedimientos y el proceso.

\section{Conclusión}

Aunque la certificación ISO 9001 presenta mejoras en algunos aspectos, según algunos estudios presentados en este artículo, se observa - en el caso estudiado- que al medir la calidad con respecto a defectos encontrados en el proceso y procedimientos, éstos no presentaron calidad de competencia mundial, A pesar de estar certificados con ISO 9001, lo cual indica que se deben implementar técnicas que mejoren la calidad de los procesos de la organización - aun cuando los procesos estén controlados por la norma ISO 9001- y fortalezcan la incidencia en la calidad de la implementación de la norma ISO 9001.

\section{Referencias}

Anderson, S.; Daly, D.; Johnson, M. (1999). Why Firms Seek ISO 9000 Certification: Regulatory Compliance or Competitive Advantage? Production and Operations Management, 8(1), enero-julio, pp. 28- 43.

Andrea, C. (2016). Strategies for improving performance in the Italian local government organizations. International Journal of Quality \& Reliability Management, 33(3), julio-diciembre, pp. 344-360.

Bayati, A.; Taghavi, A. (2007). The impacts of acquiring ISO 9000 certification on the performance of SMEs in Tehran. The TQM Magazine, 19(2), julio-diceimbre, pp.140 - 149.

Benzaquen-de las Casas, J.; Convers-Sorza, J.D. (2015). El ISO 9001 y TQM en las empresas de Colombia, 9(3), septiembre, pp. 107 - 128.

Bohórquez, L.H. (2010). Estudio del impacto de la certificación ISO 9001:2000 en la competitividad-país, Ingeniería, 15(1), julio-diciembre, pp. 22 - 34.

Bondarenko-Pisemskaya, N. (2007). Acerca de las definiciones de la calidad de la educación. Revista Educere, 11(39), diciembre, pp. $613-621$.
Camisón, C.; Cruz, S.; González, T. (2006). Gestión de la calidad: conceptos, enfoques, modelos y sistemas, Pearson Educación, S.A., Madrid, pp. 840-873.

Casadesús, G.; Marti, G.; Heras, I. (2001). Benefits of ISO 9000 implementation in Spanish industry. European business review. 13(6), julio-diciembre, pp. 327-334.

Centro Nacional de Productividad, (s.f.). Impacto de la certificación de sistemas de gestión de la calidad en empresas colombianas 2000-2005.Cali: Centro Nacional de Productividad.

Escalante-Vázquez E.J. (2005). Seis Sigma. Metodologías y Técnicas, México D.F., México: Limusa, pp. 239 - 244

Fajardo-Ortiz, M.; Cano-Velasco, L.; Tobón-Gordillo, M. (2012). Impacto de la gestión de calidad en la estructura organizativa y en la innovación de la industria azucarera colombiana. Estudios Gerenciales, 28, junio, pp. 317 - 338 .

Gracia-Villar S.; Dzul-López, L.A. (2007). Modelo PEF de costes de la calidad como herramienta de gestión en empresas constructoras: una visión actual. Revista ingeniería de Construcción, 22(1), marzo, pp. 43 - 56.

Goedhuys, M; Sleuwaegen, L. (2013). The Impact of International Standards Certification on the Performance of Firms in Less Developed Countries. World Development, 47, julio, pp. 87-101.

Hernández-Sampieri, R.; Fernández-Collado, C.; BaptistaLucio, P. (1996) Metodología de la investigación, Mcgraw - Hill interamericana de México s.a. de c.v., Edo de México.

Heras-Saizarbitoriaa, I.; Aranaa, G.; Boiral, O. (2015). ¿Do ISO 9001-certified hotels get a higher customer rating thannon-certified ones? International Journal of Hospitality Management, 51, octubre, pp. 138 - 146.

Heras-Saizarbitoria, I.; Borail, O. (2015). Symbolic Adoption of ISO 9000 in SMEs: the Role of Internal Contingencies. International Small Business Journal, 33(3), julio, pp. 299-320.

Hill, S. (1996). ISO Certification Provides Long-Term Payoff. Quality, 35, enero-julio pp. 66-68.

Hurtado, R.; Rodríguez, R.; Fuentes, H.; Galleguillos, C. (2009). Impacto en los beneficios de la implementación de las normas de calidad ISO 9000 en las empresas. Revista de la facultad de Ingeniería, 23, julio-diciembre, pp.17-26.

Hong, J.; y Phitayawejwiwat, S. (2005). The impact of ISO 9000 certification on quality management practices 
in Thailand. Journal of industrial technology, 21, enero, pp. 1-6.

International Organization for Standarization, (s.f.). Gestión de la Calidad. ISO 9000:2015. Ginebra: ISO.

International Organization for Standarization, (s.f.). Management Systems Standards. [excell] Ginebra: International Organization for Standarization. Disponible en: http://isotc.iso.org/livelink/livelink?fu nc $=$ ll\&objId=18808772\&objAction=browse \&viewT ype $=1$ [Consultado el 1 de abril de 2016].

Joubert, B. (1998). ISO 9000: International Quality Standars. Production and Inventory Management Journal, 39, julio-diciembre, pp. 60- 65.

Kazez, R. (2009). Los estudios de casos y el problema de la selección de la muestra: Aportes del sistema de matrices de datos. Subjetividad y procesos cognitivos, 13(1), enero-junio, pp. 71-89. [Online] Disponible en: <http://www.scielo.org.ar/scielo. php?script=sci_arttext\&pid=S1852- [Consultado 15 de julio 2016].

Mari-Mutt, J. (2013). Manual de redacción científica [ebook], Lima: Edicionesdigitales.org. Disponible en: http://archive.org/details/ManualDeRedaccionCientifica [Consultado el 15 de mayo de 2016].

Morelos-Gómez, j.; Fontalvo, T.; Vergara, T. (2013). Incidencia de la certificación ISO 9000 en los indicadores de productividad y utilidad financiera de empresas de la zona industrial del Mamonal en Cartagena. Estudios Gerenciales, 29, enero-marzo, pp. 99-109.

Ming-Ong, O.; Kathawala, Y.; Sawalha, N. (2015). A Model for ISO9000 Quality Management System Maintenance. Quality Management Journal, 22(2), abril, pp. 5-23.

Morris, A.; Crawford, J.; Carter, D.; Mazotta, F. (2000) Management decisions for effective ISO 9000 accreditation. Management Decision, 38, enero-julio pp. 3-5.

O'Rourke, P. (2005) A multiple-case analysis of Lean Six Sigma Deployment and Implementation Strategies. Tésis de Maestría, Estados Unidos, Department of logistic of Management, Air Force institute of Technology, 176 pp. Disponible en: http://www.dtic. mil/get-tr-doc/pdf?AD=ADA437541 [Consultado 12 de enero de 2016].

Osorio-Gómez, J.C.; Cruz-Giraldo, E.V.; Romero-Vega, M.C. (2015). Impacto de la certificación ISO 9001 en clínicas de Cali, Colombia. Revista Innovar Journal Re- vista de Ciencias Administrativas y Sociales, 26(59), julio, pp. 35-46.

Robles-Marín, V. (2014). Estudio para la medición del impacto de la implementación de sistemas de gestión de calidad bajo el estándar NTC ISO 9001:2008 en las empresas de los diferentes sectores económicos de Barrancabermeja y su área de influencia. Revista Citesca, 4(7), enero, pp. 29 - 48.

Rodríguez-Fonseca, F. (2012). Incidencia de la norma ISO 9000 en Colombia y en algunas empresas en el mundo. Revista Ciencias Estratégicas. 20(27), enero-junio, pp. 149 - 160.

Rodríguez-Escobar, J.A.; González-Benito, J. (2003). Un estudio de la insatisfacción generada por la certificación ISO 9000 en la pequeña empresa. XIII Congreso Nacional de ACEDE, Salamanca, España, noviembre 2003.- Disponible en: https://gredos.usal. es/jspui/handle/10366/75270 [Consultado 20 de mayo de 2015].

Storz, C. (2007). Compliance with international standards: the EDIFACT and ISO 9000 standards in Japan social science. Social Sciences Japan Journal, 10(2), octubre, pp. 217-41.

Sumaedi S.; Yarmen, M. (2015). The Effectiveness of ISO 9001 Implementation in Food Manufacturing Companies: A Proposed Measurement Instrument. Procedia Food Science, 3, julio-diciembre, pp. 436-444.

Terziovski, M.; Samson, D.; Dow, D. (1997). The Business Value of Quality Management Systems Certification. Evidence from Australia and New Zeland. Journal of Operations Management, 15, febrero, pp. 1-18.

Wayhan, V.; Kirche, E.; Khumawhala, B. (2002). ISO 9000 Certification: The Financial Perfomance Implications. TQM, 13(2), marzo, pp. 217-231.

Zhu, Z.; Sheuermann, L. (1999). A Comparison of Quality Programs: Total Quality Management and ISO 9000. TQM, 10(2), julio-diciembre, pp. 291- 297.

\section{PARA CITAR ESTE ARTÍCULO / TO REFERENCE THIS ARTICLE / PARA CITAR ESTE ARTIGO /}

Delgado-Moreno, F.N.; Rueda Forero, P. (2019). Medición de la calidad por medio de Niveles Sigma para monitorear el mejoramiento de procesos organizacionales controlados por ISO 9001. Revista EIA, 16(31), enero-junio, pp. 225-239. [Online]. Disponible en: https://doi.org/10.24050/reia. v16i31.1113 